\title{
Generalized pairing strategies-a bridge from pairing strategies to colorings
}

\author{
Lajos Györffy \\ Bolyai Institute, \\ University of Szeged \\ email: lgyorffy@math.u-szeged.hu
}

\author{
András Pluhár \\ Department of Computer Science, \\ University of Szeged \\ email: pluhar@inf .u-szeged.hu
}

\begin{abstract}
In this paper we define a bridge between pairings and colorings of the hypergraphs by introducing a generalization of pairs called $t$-cakes for $t \in \mathbb{N}, t \geq 2$. For $t=2$ the 2 -cakes are the same as the wellknown pairs of system of distinct representatives, that can be turned to pairing strategies in Maker-Breaker hypergraph games, see Hales and Jewett [12]. The two-colorings are the other extremity of t-cakes, in which the whole ground set of the hypergraph is one big cake that we divide into two parts (color classes). Starting from the pairings (2-cake placement) and two-colorings we define the generalized t-cake placements where we pair $p$ elements by $q$ elements $(p, q \in \mathbb{N}, 1 \leq p, q<t, p+q=t)$.

The method also gives bounds on the condition of winnings in certain biased Chooser-Picker games, which can be introduced similarly to Beck [3]. We illustrate these ideas on the k-in-a-row games for different values of $k$ on the infinite chessboard.
\end{abstract}

\section{Introduction}

\section{Pairings and colorings}

Let us start by recalling pairings and two-colorings of hypergraphs. Given a hypergraph $\mathcal{H}=(\mathrm{V}, \mathrm{E})$, where $\mathrm{V}=\mathrm{V}(\mathcal{H})$ and $\mathrm{E}=\mathrm{E}(\mathcal{H}) \subseteq \mathcal{P}(\mathcal{H})=\{\mathrm{S}: \mathrm{S} \subseteq \mathrm{V}\}$

2010 Mathematics Subject Classification: 05C65, 05C15

Key words and phrases: positional games, Chooser-Picker games, k-in-a-row game, pairing strategies, hypergraph colorings 
are the set of vertices and edges, respectively. A bijection $\rho: X \rightarrow Y$, where $\mathrm{X}, \mathrm{Y} \subset \mathrm{V}(\mathcal{H})$ and $\mathrm{X} \cap \mathrm{Y}=\emptyset$ is a pairing on the hypergraph $\mathcal{H}$. An $(x, \rho(x))$ pair blocks an $A \in E(\mathcal{H})$ edge, if $A$ contains both elements of the pair. If the pairs of $\rho$ block all $A \in E(\mathcal{H})$ edges, we say that $\rho$ is a good pairing of $\mathcal{H}$.

Another, somehow related fundamental notion of hypergraphs are the twocolorings. A two-coloring of the hypergraph $\mathcal{H}=(\mathrm{V}, \mathrm{E})$ is a partition of $\mathrm{V}$ into two disjoint (color) classes. We can also define blocking in the following way. A two-coloring of $\mathcal{H}$ blocks an $A \in E$ edge, if $A$ has a non-empty intersection with both color classes. A two-coloring is a good two-coloring, if it blocks all $A \in E$ edges. Now, we underline a close relation between pairings and two-colorings that is important for us.
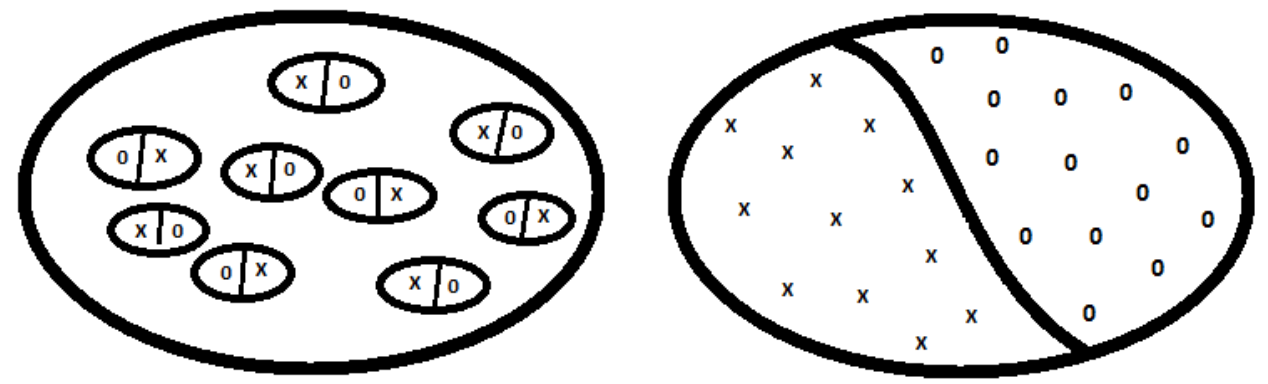

Figure 1: Pairings and two-colorings

\section{Main connection between pairings and two-colorings}

Note that a pairing induces a family of two-coloring of the hypergraph in the following sense. Instead of coloring all vertices in one step, we only color a pair in each step, one element by color 1 , the other by color 2 , until all pairs are colored (see Figure 1). The colorings of different pairs are independent. Finally we color unmatched vertices arbitrarily. If the initial pairing is good, then our coloring procedure is guaranteed to produce a good two-coloring.

From the other side, instead of pairing one vertex by one vertex, we pair a subset of vertices (color class 1) by another subset of vertices (color class 2).

Our main goal is to extend this relation into larger sets, namely when not pairs of elements, but pairs of subsets of vertices are given. We will call these pairs cakes, as these are motivated by the various cake-cutting problems. 


\section{A generalization of pairs and pairings}

Let us call a subset of the hypergraph $\mathcal{H}=(\mathrm{V}, \mathrm{E}) \mathbf{t}$-cake if it consists of exactly $\mathrm{t}$ vertices of $\mathcal{H}$, with a previously given bipartition $p, q$ of its elements, where $\mathrm{t} \in \mathbb{N}, \mathrm{t} \geq 2$ and $1 \leq \mathrm{p}, \mathrm{q} \in \mathbb{N}, \mathrm{p}, \mathrm{q}<\mathrm{t}, \mathrm{p}+\mathrm{q}=\mathrm{t}$. A $\mathrm{t}$-cake is balanced if the two parts contain equal number of elements, i. e. $p=q$.

In case of infinite hypergraphs, for example, where the vertices are the squares of the infinite chessboard, $t$ can be infinitely large, too.

Definition 1 A $t$-cake placement $\mathcal{T}$, briefly a t-placement, on the $\mathcal{H}=$ ( $\mathrm{V}, \mathrm{E})$ hypergraph is a non-overlapping placement of cakes on the hypergraph, where the size of every cake is at most $\mathrm{t}$. If a $\mathrm{t}$-placement for an even $\mathrm{t}$ contains only balanced $\mathrm{t}$-cakes, we are talking about $\boldsymbol{p}$-pairing, where $\mathrm{p}=\mathrm{t} / 2$.

In a $t$-placement, the different cakes can have different sized parts, so $p$ depends on the actual cake. However, in most of our examples we deal with only p-pairings.

Clearly, a pairing is a 1-pairing and also a 2-placement $(t=2, p=1)$. In fact, any pair has a unique way to be viewed as a cake. A two-coloring is also a special $t$-placement, where $t=|\mathrm{V}|$ and the parts of the cake are the two color classes. Similarly to pairings and colorings, the concept of blocking is also crucial for $\mathrm{t}$-placements.

Definition 2 For a hypergraph $\mathcal{H}=(\mathrm{V}, \mathrm{E})$ a $\mathrm{t}$-cake blocks an $\mathrm{A} \in \mathrm{E}$, if both parts of the cake have a non-empty intersection with A. A t-placement $\mathcal{T}$ is a good $\boldsymbol{t}$-placement of $\mathcal{H}$, if all edges of $\mathcal{H}$ are blocked by a cake of $\mathcal{T}$. An $\mathrm{A} \in \mathrm{E}$ of $\mathcal{H}$ is an unblocked edge, if there is no cake in $\mathcal{T}$ blocks $\mathrm{A}$.

We see a kind of monotonicity: if there is a good t-placement for a hypergraph $\mathcal{H}$, then there is a good $(t+1)$-placement for $\mathcal{H}$, by definition.

Similarly to pairings, $t$-placements can be considered as a step by step coloring, too. Instead of coloring a pair (as by pairings), we color a cake in each step, one part of the cake by color 1 and the other one by color 2 . In the case of a good t-placement, the step by step coloring produces a good coloring.

Let us recall now some definitions of hypergraph games.

\section{Hypergraph games}

We can define several games on a given hypergraph $\mathcal{H}=(\mathrm{V}, \mathrm{E})$, where $\mathrm{V}$ can be infinite, but an $A \in E$ edge is always finite. The first and second players 
take elements of $\mathrm{V}$ in turns. In the Maker-Maker (M-M) version of the game, the player who is first to take all elements of some edge $A \in E$ wins the game. In the Maker-Breaker (M-B) version, Maker wins by taking every element of some $A \in E$. The other (usually the second) player, Breaker, wins by taking at least one vertex of every edge in $\mathrm{E}$. Clearly, there is no draw in this game. The M-M and M-B games are closely related, since if Breaker wins as a second player, then the M-M game is a draw. On the other hand, if the first player has a winning strategy for the M-M game, then Maker also wins the M-B version. For more on these, see Berlekamp, Conway and Guy [5] or Beck [4].

There are biased $(p, q)$ M-B games, where Maker takes $p$ and Breaker takes q elements in each turn. In Chvátal and Erdős [6], Hefetz et al. [13], Krivelevich [14] and Pluhár [15] one can find more examples and applications of biased games.

Beck introduced Picker-Chooser and Chooser-Picker versions of M-B games in Beck [2], where the two players are Picker and Chooser. Picker selects a pair of elements, neither of which had been selected previously, and Chooser keeps one of these elements and gives the other one back to Picker. In the Chooser-Picker (C-P) version Chooser plays as Maker and Picker plays as Breaker, while the roles are swapped in the Picker-Chooser (P-C) game.

Similarly to the M-B games, we define a possible version of the biased Chooser-Picker game. Note that another version is used by Csernenszky [8].

Definition 3 In the biased(t) Chooser-Picker games Picker as Breaker takes maximum $t$ elements instead of one pair, and divides this set into two nonempty, disjoint parts. Chooser keeps one of these parts and gives the other part back to Picker.

Note that the results on the t-placements give winning conditions on the biased( $\mathrm{t}$ ) C-P games right away.

Since in our paper the k-in-a-row type games play an important role, we define $\mathcal{H}_{k}$, the hypergraph of the k-in-a-row games.

Definition 4 The vertices of the $\boldsymbol{k}$-in-a-row hypergraph $\mathcal{H}_{\mathrm{k}}$ are the squares of the infinite (chess)board, i. e. the infinite square grid. The edges of the hypergraph $\mathcal{H}_{\mathrm{k}}$ are the $\mathrm{k}$-element sets of consecutive squares in a row horizontally, vertically or diagonally.

Obviously, a good t-placement for $\mathcal{H}_{k}$ is a good t-placement for $\mathcal{H}_{k+1}$, too. 


\section{Some facts about pairings and t-placements}

\section{$2.1 \quad$ Pairings}

Pairings are one way to show that Breaker has a winning strategy in hypergraph games. A good pairing $\rho$ for a hypergraph $\mathcal{H}$ can be turned to a winning pairing strategy for Breaker in the $\mathrm{M}-\mathrm{B}$ game on $\mathcal{H}$. Following $\rho$ on $\mathcal{H}$ in a M-B game, for every $x \in X \cup Y$ element chosen by Maker, Breaker chooses $\rho(x)$ or in case of $x \in Y$ vice versa. If $x \notin X \cup Y$ then Breaker can choose an arbitrary vertex. Hence Breaker can block all edges and wins the game. Since we will study the $\mathcal{H}_{k}$ hypergraphs, our illustration is the k-in-a-row Maker-Breaker game.

The best upper bound for the value of $k$, where Breaker has a winning strategy in the k-in-a-row game is given by T. G. L. Zetters (alias A. Brouwer) [11]. He showed that Breaker can win the 8-in-a-row game. The best lower bound is given by Allis [1], he has shown that Maker wins the 5-in-a-row game on the boards of sizes $19 \times 19$ and $15 \times 15$. The following result is due to Hales and Jewett [5], which yields only a little weaker upper bound:

Theorem 1 Breaker wins the 9-in-a-row $M-B$ game by a pairing strategy, $i$. e. there exists a good pairing for the 9-in-a-row.

Proof. Figure 2 is an extension of a pairing of an $8 \times 8$ torus (framed), where the pairs have a periodicity 8 in every line. Hence, the pairing blocks all 9-ina-row edges.

A pairing is a domino pairing on the square grid, if all pairs consist of neighboring cells (horizontally, vertically or diagonally). Note that the pairing on Figure 2 is a domino pairing.

Csernenszky et al. [9] showed that to decide whether there exists a pairing strategy for an arbitrary hypergraph, is an NP-complete problem. A counting type proposition showed [9] that there is no good pairing strategy for $\mathcal{H}_{k}$, if $k<9$. Our main purpose to introduce the notion of $t$-placement was to extend the pairing strategy to a wider class of hypergraph games (for example $\mathcal{H}_{k}$ where $k<9$ ). Since the previous proposition plays an important role in our discussion, we formulate the exact statement.

For a hypergraph $\mathcal{H}$ let $\mathrm{d}_{2}(\mathcal{H})$ (briefly $\mathrm{d}_{2}$ ) be the greatest number, that many edges can be blocked by two vertices of $\mathcal{H}$, i. e. $d_{2}$ is the maximal co-degree.

Proposition 1 [9] If there is a good pairing $\rho$ for the hypergraph $\mathcal{H}=(\mathrm{V}, \mathrm{E})$, then $\mathrm{d}_{2}|\mathrm{X}| / 2 \geq|\mathcal{G}|$ must hold for all $\mathrm{X} \subset \mathrm{V}$, where $\mathcal{G}=\{\mathrm{A}: \mathrm{A} \in \mathrm{E}, \mathrm{A} \subset \mathrm{X}\}$. 


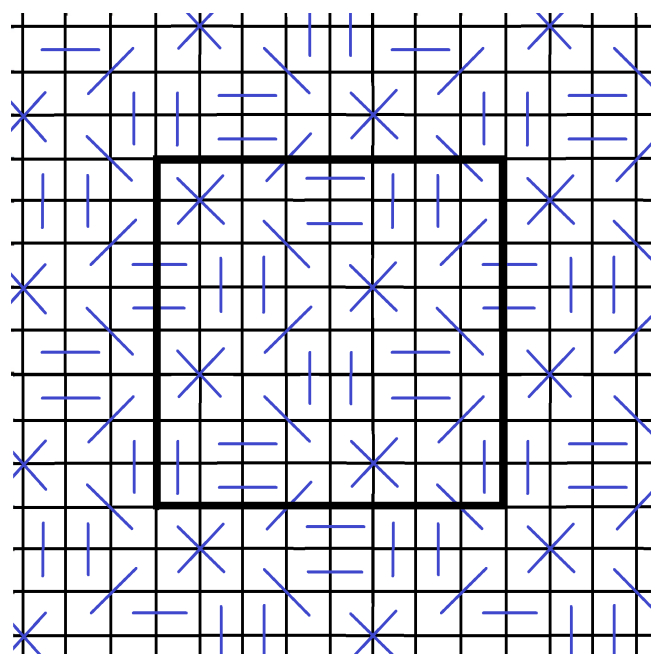

Figure 2: Hales-Jewett pairing against 9-in-a-row (Berlekamp et al. [5])

Proof. We will refer to $X$ as a subboard. The edges of $\mathcal{G}$ can be blocked only by pairs coming from $X$. There are at most $|X| / 2$ such pairs of $\rho$ on the subboard of size $|X|$. Since a pair blocks maximum $d_{2}$ edges, $|X| / 2$ pairs can block maximum $d_{2}|X| / 2$. So, if there are more edges on the subboard, there can not be a good pairing.

With the help of Proposition 1 we can conclude that there is no pairing strategy for $\mathcal{H}_{k}$ if $k<9$. In the hypergraph $\mathcal{H}_{k}, d_{2}=k-1$, because a pair can block at most $k-1$ edges. This can happen if and only if the pair is a domino. If $X$ is an $n \times n$ subboard for sufficiently large $n$, then $|\mathcal{G}|=4 n^{2}+O(n)$ because from every square four edges start (a vertical, a horizontal and two diagonal, except of the boarders). By Proposition 1, we have $(k-1) n^{2} / 2 \geq 4 n^{2}+O(n)$; that is, $k \geq 9+O(1 / n)$.

\section{2 t-placements}

As pairings can help Breaker in the M-B and Picker in the normal C-P games, $\mathrm{t}$-cakes can also help Picker in the biased( $\mathrm{t}$ ) C-P games. If there is a good $\mathrm{t}$-placement blocking a hypergraph, then for the corresponding biased( $\mathrm{t}$ ) C-P hypergraph game Picker as Breaker has a winning strategy by just giving the cakes to Chooser in any order.

Let us count the number of hypergraph edges can be blocked by a given cake, that is the blocking number of the cake. In order to generalize the 
co-degree argument, we also have to take into consideration the shape and bipartition of the cake. For a given $t \in \mathbb{N}$ let $d_{t}$ be the greatest blocking number among all $t$-cakes. A $t$-cake with blocking number $d_{t}$ is called a best $t$-cake. Note that $d_{t}$ is a monotonic function of $t$, because if a $t$-cake blocks $d_{t}$ edges in a hypergraph, then adding any part by a single vertex the obtained $(t+1)$-cake blocks all previously blocked edges, too. However, it is easy to see that the ratio $d_{t} / t$ - the best average blocking number per vertex - is not necessarily monotonic in $t$. Still, a case analysis shows that for the $\mathcal{H}_{k}$ hypergraph that the ratio $d_{t} / t$ is monotonic in $t$ at least if $t \leq 8$, as we will see later (for $t \geq 9$ it is undecided yet). With all these we can spell out the following generalization of Proposition 1.

Proposition 2 If there is a good $\mathrm{t}$-placement of $\mathcal{H}=(\mathrm{V}, \mathrm{E})$ such that $\mathrm{d}_{2} / 2 \leq$ $\mathrm{d}_{3} / 3 \leq \cdots \leq \mathrm{d}_{\mathrm{t}} / \mathrm{t}$, then $\frac{\mathrm{d}_{\mathrm{t}}}{\mathrm{t}}|\mathrm{X}| \geq|\mathcal{G}|$ for every $\mathrm{X} \subset \mathrm{V}$, where $\mathcal{G}=\{\mathrm{A}: \mathrm{A} \in$ $\mathrm{E}, \mathrm{A} \subset \mathrm{X}\}$.

From now on we assume that $\mathrm{V}$ is the set of squares of the infintie square grid. In this case a t-cake has geometrical shape. In the next section we consider some possible small $t$-cakes for $\mathcal{H}_{k}$, and we will list the best $t$-cakes for all $t \leq 8$.

\section{Some t-cakes and the maximal blocking numbers}

The most interesting and treatable examples are the 4-cakes, we start with those.

\subsection{4 -cakes in general}

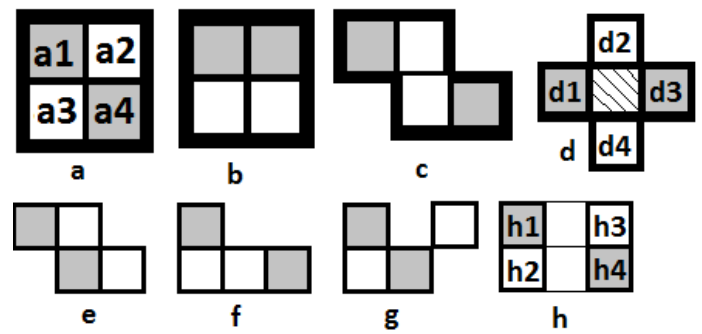

Figure 3: Some 4-cakes

On Figure 3 there are some 4-cakes. The partitions of the cakes are the white and gray squares. In the first row on Figure 3, all 4 -cakes block $4 k-4$ 
edges of the k-in-a-row. However, the cakes in the second row block fewer than $4 k-4$ edges.

For example, 4-cake a blocks in two vertical and two horizontal directions: along the lines $(a 1, a 3)$ and $(a 2, a 4)$ vertically, while along $(a 1, a 2)$ and ( $a 3, a 4)$ horizontally. In detail, $(a 1, a 3)$ blocks $k-1$ edges in its direction as a single domino, because $a 1$ and $a 3$ are neighboring cells and they are in different parts of the cake $a$. The same is true for the pairs (a2, a4), (a1, a2) and ( $a 3, a 4)$. Adding up, the cake a blocks $4(k-1)$ edges. The pairs $(a 1, a 4)$ and $(a 2, a 3)$ do not block any k-in-a-row edges because those are in the same parts of the cake $a$, hence $a$ does not block any diagonal edges.

Cakes b and c (or their rotated copies) block edges on two diagonal and on two horizontal (or rotatedly vertical) lines, while $d$ (which does not contain the central square) blocks in four diagonal directions. Since on each line there are neighboring cells (dominoes) blocking, those block $k-1$ edges per line, which adds up $4(k-1)$.

Cakes $e$ and $f$ block only in three directions, adding up only to $3(k-1)$ edges. The cakes $g$ and $h$ block in four directions, but not only by neighboring cells. Obviously, cake $g$ blocks $4 k-5$ and cake $h$ blocks $4 k-6$ edges.

\subsection{The maximal blocking numbers of $\mathrm{t}$-cakes for $2 \leq \mathrm{t} \leq \mathbf{8}$}

The 2-cakes are the pairs. One pair blocks maximum $k-1$ edges of the $k$ in-a-row. Hence, the edges of $\mathcal{H}_{k}$ can be blocked by pairs only for $k \geq 9$, see Proposition 1.

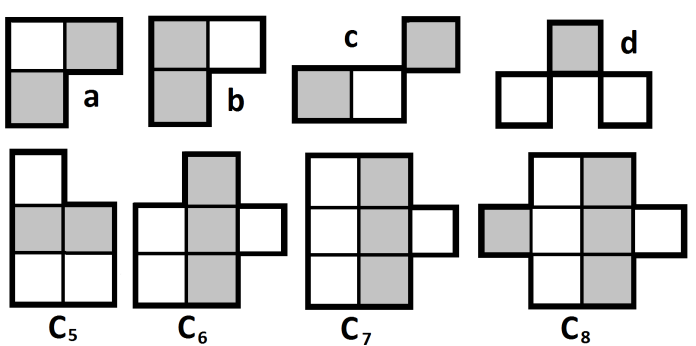

Figure 4: The best 3-cakes (first row) and the best 5-, 6-, 7- and 8-cakes

It is easy to see that a 3 -cake (with a $1+2$ bipartition) cannot block more than $2 k-2$ edges of $\mathcal{H}_{k}$. On the first row of Figure 4 we listed all best 3-cakes. 3 -cake a blocks $(k-1)+(k-1)$ edges in a horizontal and a vertical, $d$ in two diagonal, $\mathrm{b}$ and $\mathrm{c}$ in a diagonal and in a vertical (rotatedly in a horizontal) 
direction. Since all other 3-cakes block fewer edges than the previous cakes, $\mathrm{d}_{\mathrm{t}}=2 \mathrm{k}-2$. Applying the Proposition 2 on an $X=n \times n$ board for sufficiently large $n$, we get $(2 k-2) n^{2} / 3 \geq 4 n^{2}+O(n)$, so $k \geq 7+O(1 / n)$, hence a 3 placement cannot block $\mathcal{H}_{6}$, but theoretically it can block $\mathcal{H}_{7}$ or $\mathcal{H}_{8}$. However, it is still an open question if there exists any good 3-placement for 7- or 8-ina-row.

In the previous subsection on the first row of Figure 3 we listed 4-cakes blocking $4 k-4$ edges. We note that this is the maximum number of edges that can be blocked by any 4-cake. Actually, the first row of Figure 3 contains the complete list of the best 4 -cakes. Hence, $d_{4}=4 k-4$. Applying the Proposition 2 we get $(4 k-4) n^{2} / 4 \geq 4 n^{2}+O(n)$, so $k \geq 5+O(1 / n)$. Therefore, theoretically there can exist a good 4 -placement for $\mathcal{H}_{5}$ and $\mathcal{H}_{6}$, but these questions are undecided yet.

In the second row of the Figure 4, there are the best $t$-cakes for $5 \leq t \leq 8$, named $C_{5}, C_{6}, C_{7}$ and $C_{8}$. In the following table we summarize the values of $d_{t}, d_{t} / t$ and the lower bounds of $k$ drawn from Proposition 2, for that a t-placement can block $\mathcal{H}_{k}$.

\begin{tabular}{|c||c|c|c|c|c|c|c|}
\hline$t$ & 2 & 3 & 4 & 5 & 6 & 7 & 8 \\
\hline \hline$d_{t}$ & $k-1$ & $2 k-2$ & $4 k-4$ & $5 k-4$ & $7 k-6$ & $9 k-8$ & $11 k-9$ \\
\hline$\frac{d_{t}}{t}$ & $\frac{1}{2} k-\frac{1}{2}$ & $\frac{2}{3} k-\frac{2}{3}$ & $k-1$ & $k-\frac{4}{5}$ & $\frac{7}{6} k-1$ & $\frac{9}{7} k-\frac{8}{7}$ & $\frac{11}{8} k-\frac{9}{8}$ \\
\hline$k \geq$ & 9 & 7 & 5 & 4.8 & 4.29 & 4 & 3.73 \\
\hline
\end{tabular}

Hereafter we can spell out results on $\mathcal{H}_{k}$. Namely, for a given $k$ which is the smallest value of $t$ for that there exists a good $t$-placement for $\mathcal{H}_{k}$.

\section{4 k-in-a-row}

At first we list our results in a table. Columns and rows stand for the values of $k$ and $t$, respectively. "Yes" designates the existence of a good placement, "No" means that there is no good placement, while the case of "?" is undecided yet.

Some of the "No's" are the simple consequences of the previous propositions. The rest of the paper is devoted to prove the remaining claims which are summarized in the table. In the rest we will use $X$ and $O$ for the colors (as usually in the Tic-Tac-Toe game). 


\begin{tabular}{|c||c|c|c|c|c|c|c|c|c|}
\hline$k \backslash t$ & 2 & 3 & 4 & 5 & 6 & 7 & 8 & $t \geq 9$ & $\infty$ \\
\hline \hline 2 & No & No & No & No & No & No & No & No & No \\
\hline 3 & No & No & No & No & No & No & No & No & Yes \\
\hline 4 & No & No & No & No & No & No & No & $?$ & Yes \\
\hline 5 & No & No & $?$ & $?$ & $?$ & $?$ & Yes & Yes & Yes \\
\hline 6 & No & No & $?$ & $?$ & Yes & Yes & Yes & Yes & Yes \\
\hline 7 & No & $?$ & Yes & Yes & Yes & Yes & Yes & Yes & Yes \\
\hline 8 & No & $?$ & Yes & Yes & Yes & Yes & Yes & Yes & Yes \\
\hline 9 & Yes & Yes & Yes & Yes & Yes & Yes & Yes & Yes & Yes \\
\hline
\end{tabular}

\subsection{The case 2-in-a-row}

Remark 1 There exists no good two-coloring of the infinite board, which can block all $\mathcal{H}_{2}$ edges on the board.

Proof. Assume that we have a good coloring. Let us choose an arbitrary square having the color say $X$. If there is an other $X$ among its neighbors, we can not get a good coloring. But if all of its eight neighbors have color $\mathrm{O}$, then among the neighbors there are two monochromatic cells next to each other.

\subsection{The case 3 -in-a-row}

The next theorem is in fact a special case of the Theorem 2 in Dumitrescu and Radoičić [10]. We give the sketch of their proofs.
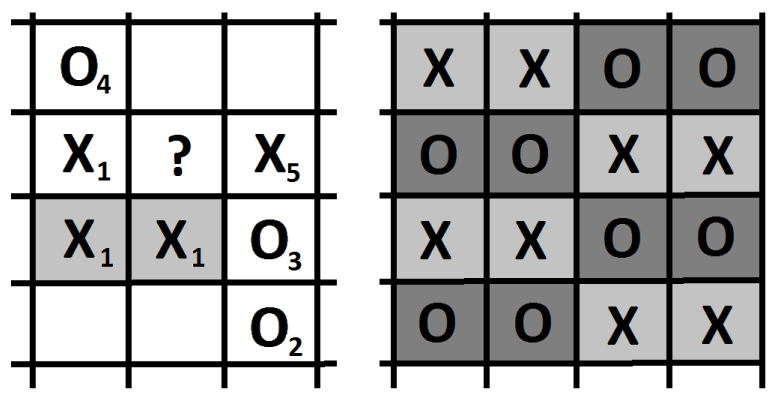

\begin{tabular}{|l|l|l|l|l|l|l|l|}
\hline$x$ & 0 & 0 & 0 & $x$ & 0 & 0 & 0 \\
\hline 0 & 0 & $x$ & 0 & 0 & 0 & $x$ & 0 \\
\hline 0 & $x$ & $x$ & $x$ & 0 & $x$ & $x$ & $x$ \\
\hline 0 & $x$ & $x$ & $x$ & 0 & $x$ & $x$ & $x$ \\
\hline$x$ & 0 & 0 & 0 & $x$ & 0 & 0 & 0 \\
\hline 0 & 0 & $x$ & 0 & 0 & 0 & $x$ & 0 \\
\hline 0 & $x$ & $x$ & $x$ & 0 & $x$ & $x$ & $x$ \\
\hline 0 & $x$ & $x$ & $x$ & 0 & $x$ & $x$ & $x$ \\
\hline
\end{tabular}

Figure 5: Colorings blocking 3- and 4-in-a-row edges

Theorem 2 There is a unique good two-coloring of the board for $\mathcal{H}_{3}$. 
Proof. Following the left hand side of the Figure 5, there are at least two edge-neighboring cells on the board, which have the same color (colored by light gray on Figure 5). If not, we would get the infinite chessboard coloring with arbitrary long monochromatic diagonal lines.

Assume the three $X$ 's with index 1 in our coloring. $\mathrm{O}_{2}, \mathrm{O}_{3}, \mathrm{O}_{4}$ and $\mathrm{X}_{5}$ are forced if we want to exclude monochromatic 3 -in-a-row edges. The square marked by an "?" gives a contradiction.

This proves that if we take two edge-neighboring X's (let us say in a row), then any square right above or under them must contain an $\mathrm{O}$. This ensures that the global coloring must be the middle coloring of Figure 5.

Corollary 1 There is no good $\mathrm{t}$-placement with finite $\mathrm{t}$ for $\mathcal{H}_{3}$.

Proof. This follows from the uniqueness of the coloring of the Theorem 2 . If there were a good $t$-placement with a finite $t$, then the colorings of some cake-parts could be switched, which would result in infinite many good colorings.

\subsection{The case 4 -in-a-row}

Of course by the monotonicity in $k$ the previous coloring of $\mathcal{H}_{3}$ blocks also $\mathcal{H}_{4}$. For $\mathcal{H}_{4}$ there is an other good two-coloring in the paper of Dumitrescu and Radoičić [10], see on the right hand side of the Figure 5. Note that this coloring blocks four consecutive monochromatic squares on all lines, including every rational slope. One may ask, how many good two-colorings are for $\mathcal{H}_{4}$ ?

It is also an open question if there is a good $t$-placement with finite $t$ for $\mathcal{H}_{4}$. From Proposition 2 we know a lower bound, that there are no good $t$ placement for $\mathcal{H}_{4}$ for $t \leq 6$. On the other hand, Observation 2 shows that there are no $t$-placement for $t=7$ and 8 , too.

Remark 2 There are no good $\mathrm{t}$-placement for $\mathrm{t}=7$ and 8 for $\mathcal{H}_{4}$.

Proof. Consider the following. For $t=7,8$ a good placement must contain at least one cake $C_{7}$ and/or $C_{8}$ of Figure 4. (Any other 7- and 8-cakes block fewer edges than it would be enough to block $\mathcal{H}_{4}$.) But $C_{7}$ and $C_{8}$ contain three consecutive squares in a vertical row in the same part of the cakes. The color of the two squares neighboring the three consecutive squares in $C_{7}$ and $\mathrm{C}_{8}$ is uncontrolled and results in an unblocked 4-in-a-row. 


\subsection{The cases 9- or more-in-a-row}

Theorem 1 shows "Yes" for $t=2, k=9$. Because of monotonicity, it also holds for every $k \geq 9$. Moreover, it follows from Proposition 1, that pairings cannot block all $k$-in-a-row edges on the board for $k<9$.

\subsection{The cases 7-in-a-row and 5-in-a-row}

Theorem 3 There is a 6-placement that blocks all 7-in-a-row edges on the board.

Proof. Let us consider the 6-placement on Figure 6, where the different parts of the cakes are colored by gray and white. That placement clearly blocks horizontally three, vertically four and diagonally seven consecutive squares in every row.

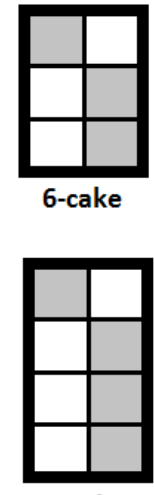

8-cake

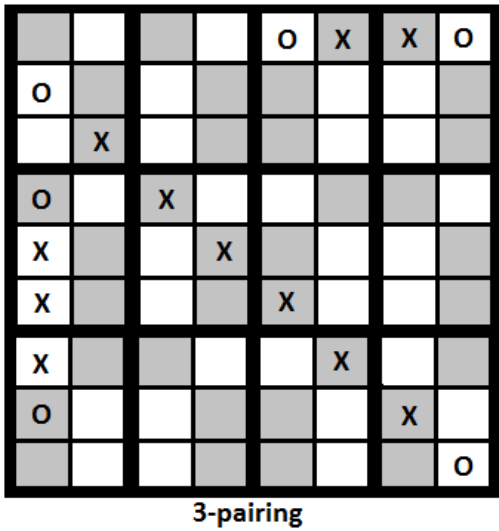

3-pairing

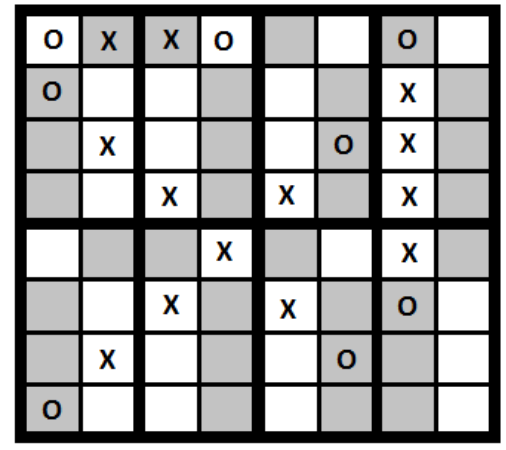

4-pairing

Figure 6: 6- and 8-cakes blocking 7- and 5-in-a-row

Theorem 4 There is an 8-placement that blocks all 5-in-a-row edges on the board.

Proof. Modifying the previous 6-cakes by two additional squares, we obtain the 8-placement on the right of Figure 6. That placement blocks all 5-in-arow edges on the board, even more, it blocks every three consecutive squares horizontally.

Corollary 2 Theorem 4 implies that Picker as Breaker wins the biased(8) C-P 5-in-a-row game. 


\subsection{The case 7-in-a-row by 4-cakes}

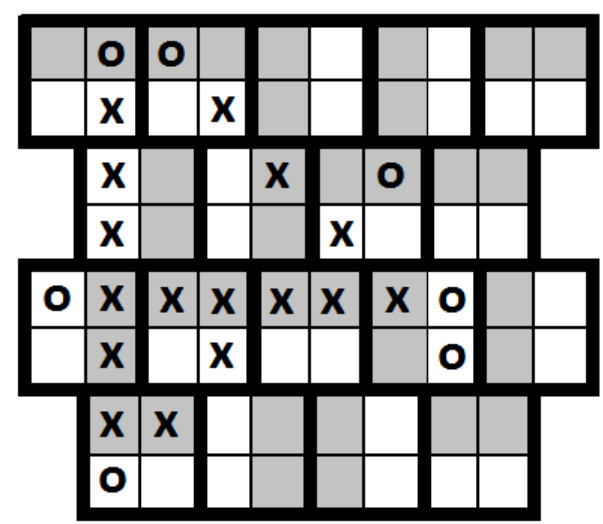

Figure 7: 2-pairing blocks 7-in-a-row edges

Theorem 5 There is a good 4-placement for the 7-in-a-row.

Proof. The 4-placement on Figure 7 blocks every 7-in-a-row edges.

Note that the same placement blocks all 8-in-a-row edges by monotonicity.

Corollary 3 Theorem 5 implies that Picker as Breaker wins the biased(4) $C$-P 7-in-a-row game.

Even more, from Csernenszky [7] we know that Picker wins the normal C-P 7-in-a-row game, too.

So far all $t$-placements we gave were also a $t / 2$-pairing by definition. Our last example for $\mathcal{H}_{6}$ shows that there are other interesting good $t$-placements.

\subsection{The case 6 -in-a-row}

Theorem 6 There is a good 6-placement for $\mathcal{H}_{6}$.

Proof. Let us consider the two types of 6-cakes placed alternately in the 6placement of Figure 8. That configuration obviously provides the appropriate blocking.

Corollary 4 Theorem 6 implies that Picker as Breaker wins the biased(6) C-P 6-in-a-row game. 

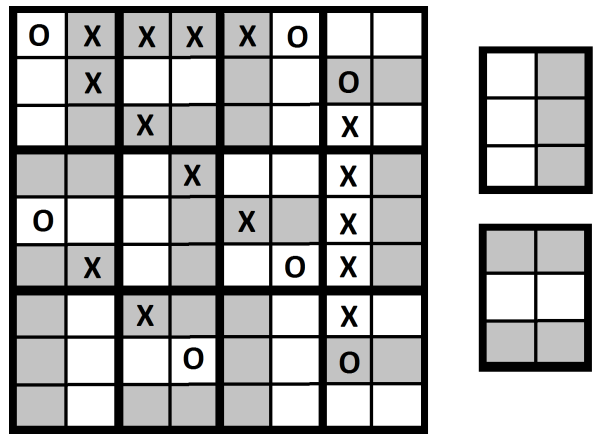

Figure 8: 6-cakes block 6-in-a-row

\section{Conclusion and open problems}

In this paper we built a bridge between the pairings and the two-colorings of hypergraphs. We have introduced a new object, the $t$-cakes, and illustrated their use in the game $k$-in-a-row for some $k \in \mathbb{N}$.

The 9-in-a-row Maker-Maker game is a draw, even more, Breaker wins the Maker-Breaker version of it by a pairing strategy. The best result is due to Brouwer (see T.G.L. Zetters [11]) who proved some 35 years ago that Breaker can win the 8-in-a-row, while Csernenszky et al. [9] showed that it cannot be done by a pairing strategy. On the other direction, Allis [1] showed that the first player wins the Maker-Maker 5-in-a-row game on the boards of sizes $19 \times 19$ and $15 \times 15$. The cases of the infinite board and $k=6$ and $k=7$ are still open.

Picker (as Breaker) wins the Chooser-Picker version of the 7 -in-a-row game (Csernenszky [7]); the cases for $k \leq 6$ are open. In the biased versions - in which Picker selects $t$ elements and divides it into two parts, then Chooser takes one of those parts - Picker wins even if $k \leq 6$.

While there are no good pairings for the 7 -in-a-row game, there is a good 2-pairing (4-placement) which is a generalization of pairings. Obviously, by monotonicity, it works for 8-in-a-row, too. In the case of 6-in-a-row, there is a good 6-placement, that means Picker as Breaker wins the biased(6) 6-in-a-row game. To win the 5-in-a-row, Picker has a good 4-pairing (8-placement). It is still open if there are good $t$-placements for $4 \leq t \leq 7$.

We think that no finite cakes can block the winning sets of the hypergraph 4 -in-row. Still, we can prove this only in the case when the sizes of the cakes are not larger than eight. There are more than one good two-colorings $(\infty-$ 
placements) blocking all 4-in-a-row edges on the board. The number and the structure of those good colorings are still open questions.

The 3 -in-a-row case is very simple, there is a unique good two-coloring. Then the biased $(\infty)$ k-in-a-row game is that Picker divides the infinite square grid into two parts, neither of those containing three consecutive squares in a row. Finally, there are no two-colorings blocking all 2-in-a-row edges.

\section{Acknowledgements}

Special thanks go to Peter Hajnal for his useful comments and his generous help.

\section{References}

[1] L. V. Allis, H. J. van den Herik and M. P. Huntjens, Go-Moku solved by new search techniques. Proc. 1993 AAAI Fall Symp. on Games: Planning and Learning, AAAI Press Tech. Report FS93-02, 1-9, Menlo Park.

[2] J. Beck, Positional games and the second moment method. Combinatorica, 22 (2002), 169-216.

[3] J. Beck, Positional Games, Combinatorics, Probability and Computing, 14 (2005), 649-696.

[4] J. Beck, Combinatorial Games, Tic-Tac-Toe Theory, Cambridge University Press 2008.

[5] E. R. Berlekamp, J. H. Conway and R. K. Guy, Winning Ways for your mathematical plays, Volume 2, Academic Press, New York 1982.

[6] V. Chvátal and P. Erdős, Biased positional games, Annals of Discrete Math., 2 (1978), 221-228.

[7] A. Csernenszky, The Chooser-Picker 7-in-a-row game. Publicationes Mathematicae, 76 (2010), 431-440.

[8] A. Csernenszky, The Picker-Chooser diameter game. Theoretical Computer Science, 411 (2010), 3757-3762.

[9] A. Csernenszky, R. Martin and A. Pluhár, On the Complexity of ChooserPicker Positional Games, Integers, 12 (2012), 427-444. 
[10] A. Dumitrescu and R. Radoičić, On a coloring problem for the integer grid, Towards a Theory of Geometric Graphs, (2004), 67-74.

[11] R. K. Guy and J. L. Selfridge, Problem S.10, Amer. Math. Monthly 86 (1979); solution T.G.L. Zetters 87 (1980), 575-576.

[12] A. W. Hales and R. I. Jewett, Regularity and positional games, Trans. Amer. Math. Soc., 106 (1963), 222-229; M.R. \# 1265.

[13] D. Hefetz, M. Mikalački and M. Stojaković, Doubly biased Maker-Breaker Connectivity game. Electronic J. Combinatorics, 19 (2012), P61.

[14] M. Krivelevich, The critical bias for the Hamiltonicity game is $(1+$ o(1))n/lnn, Journal of the American Math. Soc., 24 (2011), 125-131.

[15] A. Pluhár, The accelerated k-in-a-row game. Theoretical Comp. Science, 270 (2002), 865-875. 